\title{
Benefits of ${ }^{11} \mathrm{C}-\mathrm{methionine} \mathrm{PET} / \mathrm{MRI}$ and intraoperative fluorescence in treating hyperparathyroidism
}

\author{
Thien Vinh Luong ${ }^{1} 1$, Lars Rejnmark ${ }^{3}$, Anne Kirstine Arveschoug ${ }^{1}$, Peter Iversen ${ }^{1}$ and \\ Lars Rolighed ${ }^{2}$
}

1Department of Nuclear Medicine and PET-Centre, 2Department of Otorhinolaryngology, and ${ }^{3}$ Department of Endocrinology and Internal Medicine, Aarhus University Hospital, Aarhus, Denmark
Correspondence should be addressed to T V Luong

Email

thiluo@rm.dk

\begin{abstract}
Multiple endocrine neoplasia 1 (MEN1) is a rare genetic syndrome characterized by the manifestation of tumors in endocrine glands most often in the parathyroid gland (PG). Treatment may involve several parathyroidectomies (PTX), especially in young patients, which increases the risk of postoperative complications. We present a 16-year-old patient with a family history of MEN1 syndrome. The patient started to show biochemical signs of hyperparathyroidism (HPT) and hypercalcemia at the age of 10. One and a half years later a PTX was successfully performed with removal of the two left PGs. However, a rise in plasma parathyroid hormone and ionized calcium was observed 4 years later. Preoperative

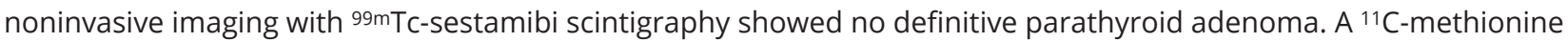
position emission tomography combined with MRI (MET-PET/MRI) was then performed and detected a focus posterior to the lower part of the right thyroid lobe. Intraoperative angiography with fluorescence and indocyanine green dye was used to assess the vascularization of the remaining PGs. The lower right PG was removed. The patient was discharged with normalized biochemical values and without postoperative complications. Recurrence of primary HPT is frequent in MEN1 patients which often necessitates repeated operations. Our case report showed that the use of advanced noninvasive preoperative imaging techniques and intraoperative fluorescent imaging are valuable tools and should be taken into consideration in selected cases to avoid postoperative complications. To our knowledge, this is the first case where METPET/MRI has been used to detect parathyroid pathology.
\end{abstract}

\section{Learning points:}

- MEN1 patients will develop parathyroid disease, which eventually will lead to surgical treatment with removal of the pathological glands.

- Preoperatively usage of MRI combined with PET tracers such as ${ }^{11} \mathrm{C}$-methionine and ${ }^{18 F}$-Fluorocholine are able to detect parathyroid pathology with a higher sensitivity than conventional imaging.

- Techniques using intraoperatively angiography with fluorescence and florescent dyes allow surgeons to verify the vascularization of each parathyroid gland.

- Optimization of noninvasive preoperative imaging techniques and intraoperative fluorescent imaging are valuable tools and should be taken into consideration when performing PTX consecutively in the same patient to avoid postoperative complications. 


\section{Introduction}

Multiple endocrine neoplasia (MEN) are rare genetic syndromes, which are characterized by the manifestation of tumors in two or more specific endocrine glands in a single patient. There exist two main forms of MEN, type 1 (MEN1) and type 2 (1). In MEN1 patients, tumors mostly occur in the parathyroid glands, the pancreatic islet cells, and in the anterior pituitary (2).The syndrome can affect all age groups with an estimated prevalence of 2 per 100000 (3) with the most common manifestation being primary hyperparathyroidism (HPT) with pathologic growth of one or more of the parathyroid glands (PG), which occurs in more than $90 \%$ of patients (1). Symptoms of HPT include abdominal pain, nausea, kidney stones, osteoporosis, depression, and tiredness. Treatment of primary HPT in MEN1 patients usually involves preoperative localization of enlarged PGs, typically with ultrasonography (US) and a 99mTc-sestamibi scintigraphy (MIBI) often combined with a single photon emission and a low-dose CT (SPECT-CT) prior to parathyroidectomy (PTX), which involves the removal of 1 or more PGs (4). The recommended surgery in MEN1 is often a subtotal PTX, where 3.5 PGs are removed. Consequently, there is a risk of developing postoperative hypoparathyroidism (hypoPT), where the remaining plasma level of parathyroid hormone (PTH) is insufficient to maintain normal plasma calcium $\left(\mathrm{Ca}^{2+}\right)$. HypoPT is a concern in young patients due to chronic need of daily medical supplementation and risks of longterm complications to hypoPT. It is, therefore, essential to precisely locate parathyroid pathology, especially because the necessity for more than one PTX is very likely in patients with MEN1.

\section{Case presentation}

We present a 16-year-old girl, who at the age of 10 in 2013 was tested positive for a mutation in the MEN1 gene established in her family. Laboratory findings revealed a slightly elevated plasma ionized calcium $\left(\mathrm{Ca}^{2+}\right)$ of 1.39 $\mathrm{mmol} / \mathrm{L}$ (reference interval 1.18-1.32 $\mathrm{mmol} / \mathrm{L}$ ) and PTH of $5.4 \mathrm{pmol} / \mathrm{L}$ (reference interval $2.3-9.3 \mathrm{pmol} / \mathrm{L}$ ). The only complaint at the time was a slight headache in the evening. After 1.5 years, the patient developed a rise in $\mathrm{Ca}^{2+}$ to $1.51 \mathrm{mmol} / \mathrm{L}$ and PTH to $11.3 \mathrm{pmol} / \mathrm{L}$, and she was referred to PTX. An US raised suspicion of an adenoma in the lower left PG. No MIBI was performed in order to avoid radiation exposure, and it was decided to remove the two left PGs. PTX was performed in 2015, and intraoperative PTH monitoring (IOPTH) showed a $74 \%$

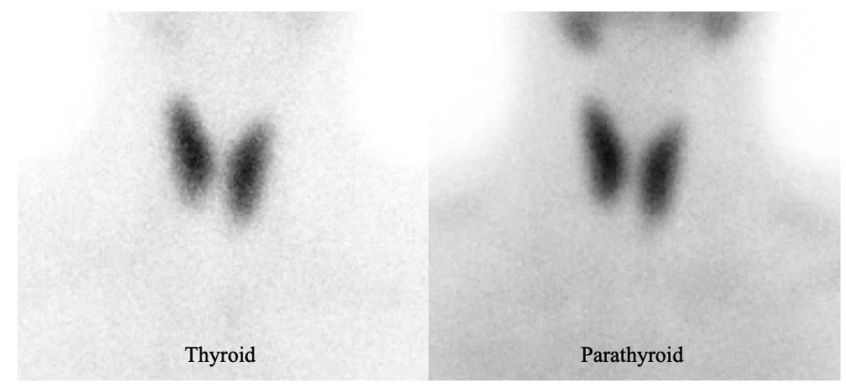

\section{Figure 1}

Planar images of the $99 \mathrm{mTC}$-sestamibi-SPECT/CT showing no clear difference between thyroid scintigraphy and parathyroid scintigraphy. In addition, no adenoma was detected on the SPECT/CT (not shown).

decrease in PTH levels after the removal of the two left glands. No postoperative complications were observed and subsequent blood test revealed a decrease in $\mathrm{Ca}^{2+}$ to $1.33 \mathrm{mmol} / \mathrm{L}$ and PTH to $3.7 \mathrm{pmol} / \mathrm{L}$.

An increase in $\mathrm{Ca}^{2+}$ was observed 4 years later and the patient was referred to a MIBI. However, no adenoma was detected (Fig. 1). Subsequently, a ${ }^{11} \mathrm{C}$-methionine position emission tomography combined with MRI (MET-PET/MR) identified a focus posterior to the lower part of the right thyroid lobe (Fig. 2). A second PTX was performed with identification of a normal upper right PG (Fig. 3) and an adenoma in the lower right capsule of the thyroid (not presented). A small distal part of the normal PG was biopsied and marked with a clip. Vascularization of the proximal remaining part of the PG was illustrated (SPY-PHI, Stryker) with intraoperative administration of $2.5 \mathrm{mg}$ Indocyanine Green (ICG) and fluorescent imaging (Fig. 4). In addition, IOPTH showed a 91\% PTH decline from $13.4 \mathrm{pmol} / \mathrm{L}$ to $1.2 \mathrm{pmol} / \mathrm{L}$. An enlarged thymus was also located on the neck and was removed with the suspicion of containing an ectopic PG, but no parathyroid tissue was detected (Fig. 5). The patient briefly experienced paresthesia in the face and right arm after the surgery and was discharged without any complications. Biochemical controls of PTH and $\mathrm{Ca}^{2+}$ were normal 2 weeks and 2 months postoperatively.

\section{Discussion}

Hyperfunctioning PGs can affect people of all age groups with MEN1 syndrome and are typically the first manifestation of the disease. It often occurs in the third decade of life with a lifelong penetrance of $100 \%$ and, therefore, international guidelines recommend extensive monitoring of these patients (1). Estimates have shown that approximately $17-23 \%$ develop primary HPT around the age of 20 years with one case as young as 8 years of age $(2,5)$. 

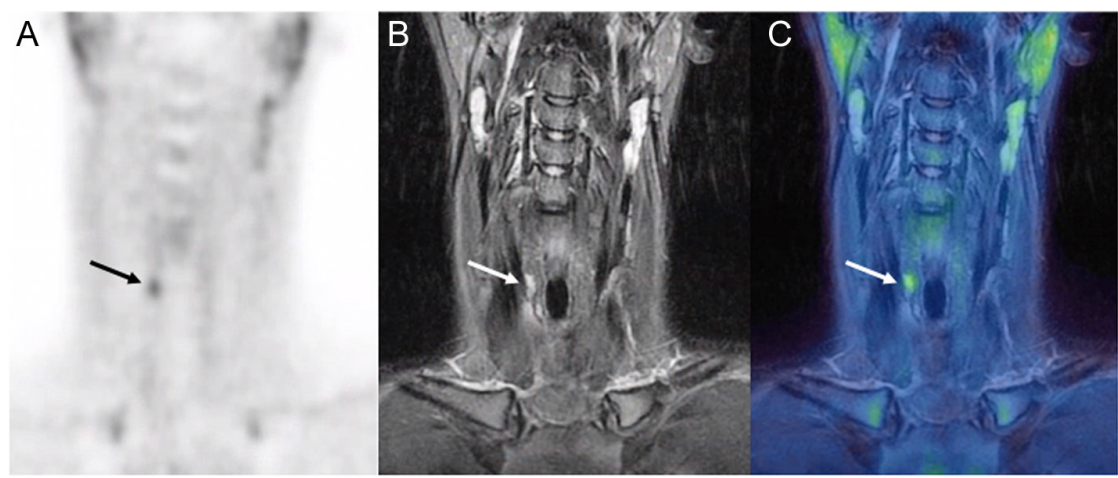

\section{Figure 2}

Frontal images of the ${ }^{11} \mathrm{C}$-methionine PET/MRI showing a focus with high ${ }^{11} \mathrm{C}$-methionine uptake (arrows) in the lower part of the right thyroid gland on the PET (A), MRI (B) and fusion image (C).
The treatment of HPT in MEN1 patients will ultimately lead to the surgical removal of abnormally overactive PGs. It is a compromise between leaving enough sufficient healthy parathyroid tissue to avoid permanent hypoPT and removing all the pathologic tissue to prevent persistence or recurrence of primary HPT, which occurs in $9-50 \%$ of cases depending on the primary surgical strategy (6). In our case, it was decided to initially perform a unilateral PTX with removal of the two left PGs to reduce the risk of affecting the recurrent laryngeal nerve and avoid the risk of developing hypoparathyroidism at a young age. At the time of the second surgery, the lower right PG as well as a small part of the upper right PG was removed, again to maintain sufficient healthy parathyroid tissue well-vascularized. Given the history of the patient, it was essential to provide a precise localization of PGs prior to the second PTX.

Noninvasive first-line imaging conducted prior to surgery usually includes US and MIBI. The sensitivities for US and MIBI range between 59-89\% and 63-84\%, respectively, which leaves a substantial amount of cases with unclear findings, necessity of more extensive surgical

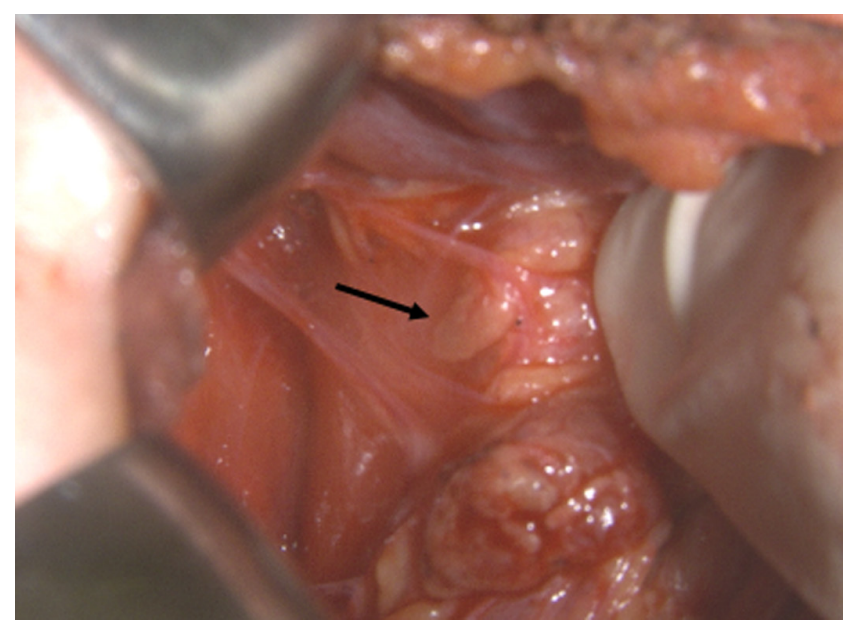

Figure 3

Right side of the neck showing a normal parathyroid gland (arrow). exploration, and a higher risk of persistent disease after surgical treatment (7). In addition, the sensitivities are low in cases with small parathyroid lesions due to the poor spatial resolution, or when the adenoma is in close proximity to the thyroid gland, and in multi gland involvement with hyperplasia (8).

Second-line noninvasive imaging usually includes CT scans, either as a 4D CT or combined with PET tracers such as ${ }^{11} \mathrm{C}$-methionine (MET) and ${ }^{18} \mathrm{~F}$-Fluorocholine (FCH) (9). In our hospital, MET-PET/CT is used in the case of negative or inconclusive conventional imaging studies before PTX or in the case of persisting HPT before a re-operation. There have been conflicting results regarding the usage of MET-PET/CT to localize pathological parathyroid glands in patients with primary HPT. However, newer studies have generally found a high sensitivity ranging from 77 to 91\% and positive predictive values (PPV) around 93-98\% $(9,10)$. In a situation where both the US and MIBI are negative, a study with 45 patients found a sensitivity of MET-PET/CT to be $74 \%$ and a PPV of $97 \%$ with a higher

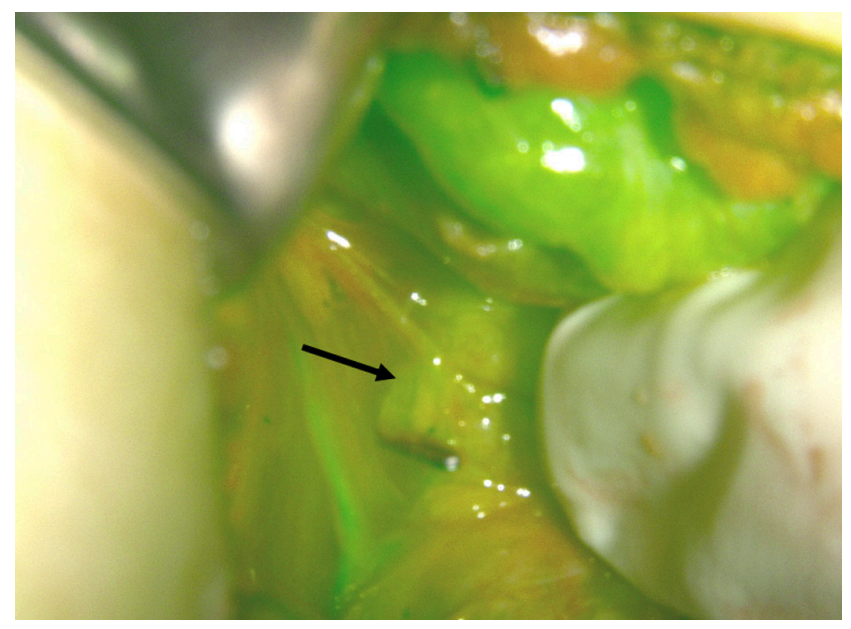

Figure 4

Usage of ICG and fluorescence with imaging of the upper right PG (arrow) marked with a clip for biopsy and future localization. 


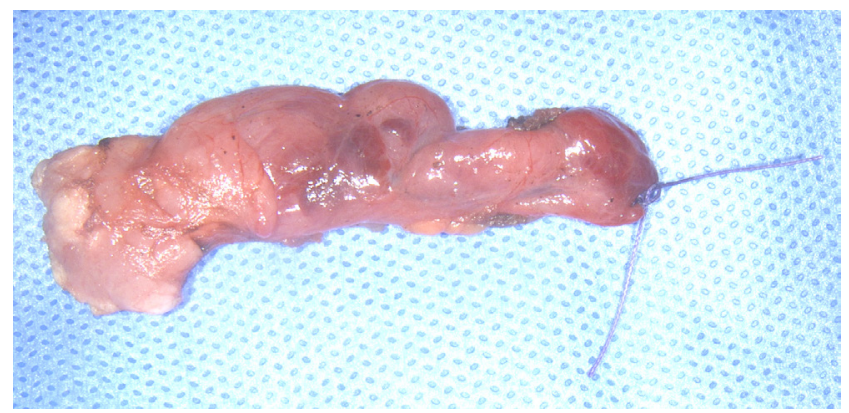

\section{Figure 5}

An enlarged right thymus was removed during the procedure with the suspicion of containing an ectopic parathyroid gland. However, no parathyroid tissue was revealed in the histologic examination.

detection rate for parathyroid adenomas (PA) compared to parathyroid hyperplasia (7). Even in the existence of a multinodular goiter or after previous neck surgery, the sensitivity and PPV values of 94\% have been found including a positive correlation with the diameter and weight of the excised PGs (10). FCH-PET combined with CT and MRI has also been shown to provide high accuracy with sensitivities and PPV values above 90\% (11).

Generally, these two PET tracers in combination with CT or MRI present themselves as highly sensitive methods to localize PAs even in atypical locations or in challenging anatomical positions in the neck, such as in patients with a multinodular goiter, or after unsuccessful PTX, and before a reoperation. They can provide valuable information, particularly for cases in which conventional imaging techniques have failed. Therefore, they are valuable tools and should be used in difficult cases, which will enable endocrine surgeons to successfully perform PTX. In our case, a MET-PET/MRI was performed to detect a PA after a negative MIBI. An MRI with a greater softtissue resolution compared with $\mathrm{CT}$, in addition to a considerably lower radiation exposure, was preferred due to the case of reoperation in a young MEN1 girl. We did not find any previous published cases where MET-PET/ MRI was used to detect parathyroid pathology.

Success of PTX heavily depends on surgeons' experience, and failure to identify an abnormal PG can lead to persistent HPT and unnecessary reoperations with an increased risk of damage to the recurrent laryngeal nerve. Furthermore, if normal functioning PGs are damaged or removed, development of hypocalcemia due to hypoPT can severely impact the patients' lives. So far IOPTH monitoring and post-operative plasma PTH levels have been used to verify the function of the remaining parathyroid tissue. Novel techniques using intraoperative angiography with fluorescence and florescent dyes are increasingly being used in the last few years. It allows surgeons to verify the vascularization of each parathyroid gland intraoperatively (12). Autofluorescence is naturally emitted from PGs and can be detected by using near-infrared fluorescence imaging. Intensity levels have been described to be greater compared to the surrounding tissue with hyperfunctioning glands displaying a more heterogeneous and lower intensity compared to normal functioning PGs (13). ICG is a fluorescent dye that displays a high uptake in well-vascularized tissue (14). Use of either autofluorescence or fluorescence with exogenic dyes have been shown to detect PGs in more than 90\% of cases, even among those with negative US and MIBI, and present themselves as valuable tools to identify and assess PGs intraoperatively $(12,13)$. However, only with the use of exogenic dyes as, for example, ICG can the vascularization for each PG be evaluated intraoperatively (Fig. 4).

\section{Conclusion}

Treatment of young MEN1 patients with manifestation of endocrine tumors is a very difficult task. It is crucial to perform a suitable treatment to enable them to live without symptoms for as long as possible. Therefore, optimization of noninvasive preoperative imaging techniques and intraoperative fluorescent imaging are valuable tools and should be taken into consideration when performing PTX repeatedly in the same patient to avoid postoperative complications. For the first time, we report the use of MET-PET/MRI as a valuable technique to detect parathyroid pathology in a challenging case.

\section{Declaration of interest}

The authors declare that there is no conflict of interest that could be perceived as prejudicing the impartiality of the research reported.

\section{Funding}

This research did not receive any specific grant from any funding agency in the public, commercial, or not-for-profit sectors.

\section{Patient consent \\ Written consent from the patient and the patient's parents have been obtained for publication of the submitted article and accompanying images.}

\section{Author contribution statement}

The manuscript was mainly written by Thien Vinh Luong and supervised by Lars Rolighed, who also performed the surgery and provided the 
fluorescence images. All listed co-authors have contributed with the scan analysis as well as proofreading of the case report.

\section{References}

1 Thakker RV, Newey PJ, Walls GV, Bilezikian J, Dralle H, Ebeling PR, Melmed S, Sakurai A, Tonelli F, Brandi ML, et al. Clinical practice guidelines for multiple endocrine neoplasia type 1 (MEN1). Journal of Clinical Endocrinology and Metabolism 201297 2990-3011. (https:// doi.org/10.1210/jc.2012-1230)

2 Kamilaris CDC \& Stratakis CA. Multiple endocrine neoplasia Type 1 (MEN1): an update and the significance of early genetic and clinical diagnosis. Frontiers in Endocrinology 201910 339. (https://doi. org/10.3389/fendo.2019.00339)

3 Trump D, Farren B, Wooding C, Pang JT, Besser GM, Buchanan KD, Edwards CR, Heath DA, Jackson CE, Jansen S, et al. Clinical studies of multiple endocrine neoplasia type 1 (MEN1). QJM 199689 653-669. (https://doi.org/10.1093/qimed/89.9.653)

4 Han CH, Fry CH, Sharma P \& Han TS. A clinical perspective of parathyroid hormone related hypercalcaemia. Reviews in Endocrine and Metabolic Disorders 202021 77-88. (https://doi.org/10.1007/ s11154-019-09529-5)

5 Herath M, Parameswaran V, Thompson M, Williams M \& Burgess J. Paediatric and young adult manifestations and outcomes of multiple endocrine neoplasia type 1. Clinical Endocrinology 201991 633-638. (https://doi.org/10.1111/cen.14067)

6 Guerin C, Paladino NC, Lowery A, Castinetti F, Taieb D \& Sebag F. Persistent and recurrent hyperparathyroidism. Updates in Surgery 201769 161-169. (https://doi.org/10.1007/s13304-017-0447-7)

7 Weber T, Gottstein M, Schwenzer S, Beer A \& Luster M. Is C-11 methionine PET/CT able to localise sestamibi-negative parathyroid adenomas? World Journal of Surgery 201741 980-985. (https://doi. org/10.1007/s00268-016-3795-4)
8 Arora S, Damle NA, Passah A, Yadav MP, Ballal S, Aggarwal V, Gupta Y, Kumar P, Tripathi M \& Bal C. Incidental detection of parathyroid adenoma on somatostatin receptor PET/CT and incremental role of ${ }^{18} \mathrm{~F}$-Fluorocholine PET/CT in MEN1 Syndrome. Nuclear Medicine and Molecular Imaging 201852 238-242. (https://doi. org/10.1007/s13139-018-0520-2)

9 Kluijfhout WP, Pasternak JD, Drake FT, Beninato T, Gosnell JE, Shen WT, Duh QY, Allen IE, Vriens MR, de Keizer B, et al. Use of PET tracers for parathyroid localization: a systematic review and metaanalysis. Langenbeck's Archives of Surgery 2016401 925-935. (https:// doi.org/10.1007/s00423-016-1425-0)

10 Weber T, Maier-Funk C, Ohlhauser D, Hillenbrand A, Cammerer G, Barth TF, Henne-Bruns D, Boehm BO, Reske SN \& Luster M. Accurate preoperative localization of parathyroid adenomas with C-11 methionine PET/CT. Annals of Surgery 2013257 1124-1128. (https:// doi.org/10.1097/SLA.0b013e318289b345)

11 Kluijfhout WP, Pasternak JD, Gosnell JE, Shen WT, Duh QY, Vriens MR, de Keizer B, Hope TA, Glastonbury CM, Pampaloni MH, et al. 18F Fluorocholine PET/MR imaging in patients with primary hyperparathyroidism and inconclusive conventional imaging: a prospective pilot study. Radiology 2017284 460-467. (https://doi. org/10.1148/radiol.2016160768)

12 Rudin AV \& Berber E. Impact of fluorescence and autofluorescence on surgical strategy in benign and malignant neck endocrine diseases. Best Practice and Research Clinical Endocrinology and Metabolism 2019 33 101311. (https://doi.org/10.1016/j.beem.2019.101311)

13 Kose E, Kahramangil B, Aydin H, Donmez M \& Berber E. Heterogeneous and low-intensity parathyroid autofluorescence: patterns suggesting hyperfunction at parathyroid exploration. Surgery 2019165 431-437. (https://doi.org/10.1016/j.surg.2018.08.006)

14 Vidal Fortuny J, Karenovics W, Triponez F \& Sadowski SM. Intraoperative indocyanine green angiography of the parathyroid gland. World Journal of Surgery 201640 2378-2381. (https://doi.org/10.1007/ s00268-016-3493-2)

Received in final form 20 April 2020

Accepted 9 July 2020 